\title{
Use of Nuclear Data Sensitivity and Uncertainty Analysis for the Design Preparation of the HCLL Breeder Blanket Mockup Experiment for ITER
}

\author{
I. Kodeli \\ OECD/NEA Data Bank, 12 boulevard des Iles, 92130 Issy-les-Moulineaux, France
}

Correspondence should be addressed to I. Kodeli, ivo.kodeli@oecd.org

Received 12 March 2008; Accepted 13 August 2008

Recommended by Igor Jencic

\begin{abstract}
An experiment on a mockup of the test blanket module based on helium-cooled lithium lead (HCLL) concept will be performed in 2008 in the Frascati Neutron Generator (FNG) in order to study neutronics characteristics of the module and the accuracy of the computational tools. With the objective to prepare and optimise the design of the mockup in the sense to provide maximum information on the state-of-the-art of the cross-section data the mockup was pre-analysed using the deterministic codes for the sensitivity/uncertainty analysis. The neutron fluxes and tritium production rate (TPR), their sensitivity to the underlying basic cross-sections, as well as the corresponding uncertainties were calculated using the deterministic transport codes (DOORS package), the sensitivity/uncertainty code package SUSD3D, and the VITAMINJ/ COVA covariance matrix libraries. The cross-section reactions with largest contribution to the uncertainty of the calculated TPR were identified to be (n,2n) and $(\mathrm{n}, 3 \mathrm{n})$ reactions on lead. The conclusions of this work support the main benchmark design and suggest some modifications and improvements. In particular this study recommends the use, as far as possible, of both natural and enriched lithium pellets for the TRP measurements. The combined use is expected to provide additional and complementary information on the sensitive cross-sections.
\end{abstract}

Copyright () 2008 I. Kodeli. This is an open access article distributed under the Creative Commons Attribution License, which permits unrestricted use, distribution, and reproduction in any medium, provided the original work is properly cited.

\section{INTRODUCTION}

The future fusion experimental reactor ITER will be based on the fusion reaction of deuterium and tritium (D-T reaction). Unlike deuterium which is stable and readily available in nature (oceans), tritium is a radioactive isotope with a relatively short half life of about 12.3 years and it will have to be produced in the fusion reactor plant locally. The tokamak will be therefore surrounded by lithium blanket and tritium will be produced by bombardment of lithium atoms with neutrons escaped from the $\mathrm{D}-\mathrm{T}$ reaction in plasma. For tritium production (breeding), two blanket concepts are considered within the EU fusion technology programme, the helium-cooled pebble bed (HCPB) blanket with lithium ceramics pebbles $\left(\mathrm{Li}_{4} \mathrm{SiO}_{4}\right.$ or $\left.\mathrm{Li}_{2} \mathrm{TiO}_{3}\right)$ as breeder material and beryllium pebbles as neutron multiplier, and the heliumcooled lithium-lead (HCLL) blanket with the Pb-Li eutectic alloy plays the role of breeder and neutron multiplier. Both blanket designs will use helium as coolant.
The two breeding-blanket modules are candidates for the blanket module design for a fusion power demonstration reactor (DEMO) to be constructed after ITER. To validate the engineering designs and the viability of the fusion plant, the test blanket modules (TBM) of both designs will be installed in ITER.

Tritium self-sufficiency which will be needed for DEMO reactor is still to be demonstrated, taking into account both the losses of the tritium inventory as well as the uncertainties in the tritium production. Experimental studies involving irradiation of the two proposed blanket designs were therefore undertaken at the Frascati Neutron Generator (FNG) $14 \mathrm{MeV}$ facility [1-3] in cooperation between the Ente per le Nuove tecnologie, l'Energia e l'Ambiente (ENEA), Frascati; Forschungszentrum Karlsruhe (FzK); Technische Universität Dresden (TUD); and Institut Jožef Stefan, Ljubljana (IJS). NEA Data Bank also participates in this study to perform transport and sensitivity studies as well as to assure the final integration of the experimental results and 


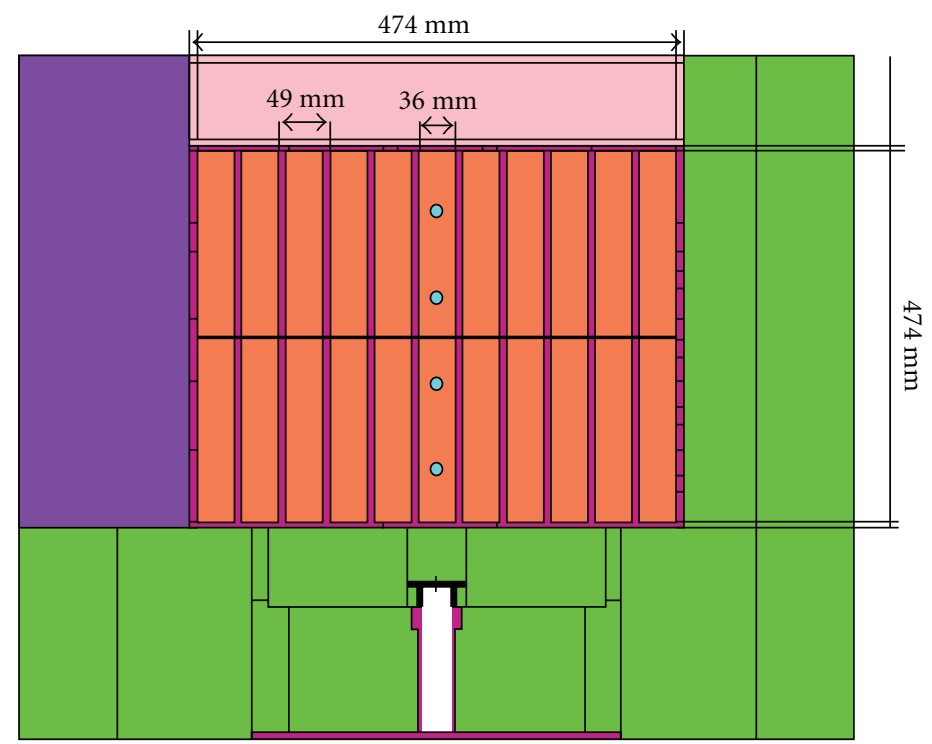

FIGURE 1: Schematic diagram of the TBM-HCLL mockup showing steel (dark red) and LiPb (orange) layers. The detectors are placed on the central axis.

their evaluation into the shielding benchmark experiments database (SINBAD) in order to make these data easily available to the international community. The objective of these experiments is to assess the uncertainty on tritium production rate (TPR) due to the uncertainty in the relevant nuclear data. The FNG experiments in particular contribute to the validation of the computational tools and nuclear data needed for the neutronics analysis. The helium-cooled pebble bed (HCPB) breeder blanket mockup benchmark experiment was performed in 2005 and consists of a metallic beryllium setup with two double layers of breeder material ( $\mathrm{Li}_{2} \mathrm{CO}_{3}$ powder). The reaction rate measurements include the $\mathrm{Li}_{2} \mathrm{CO}_{3}$ pellets for the tritium breeding monitoring and activation foils, inserted at several axial and lateral locations in the block. The experiment and the results of the analysis using the Monte Carlo and deterministic transport, sensitivity, and uncertainty code system were presented in $[4,5]$.

The tritium breeding-module helium-cooled lithiumlead benchmark experiment (TBM HCLL) is under preparation and will be performed in 2008. The aim of the present work is the preanalysis and the optimisation of the benchmark experiments design using the deterministic transport, sensitivity, and uncertainty code system. The analysis includes the calculation of the tritium production rate (TPR) in LiPb layers and the neutron reaction rates, which will be measured in the benchmark, their sensitivity to the underlying cross-sections, as well as the corresponding uncertainty estimations. The SUSD3D cross-section sensitivity and uncertainty code package together with the two- and three-dimensional $(2 \mathrm{D} / 3 \mathrm{D})$ deterministic transport codes DORT/TORT are used for the analysis of the experiment. Based on the sensitivity analyses, the most important nuclear reactions and energy ranges in the particular reaction rate measurements can be identified. This information will be used to anticipate the benefits obtained from the experiment and thus guide and optimise its design and to assess the nuclear data needs.

\section{TBM-HCLL EXPERIMENTAL MOCKUP}

The experimental geometry which will be irradiated in the FNG facility consists of a block of $45 \mathrm{~cm} \times 47.4 \mathrm{~cm}$ side view and $34.6 \mathrm{~cm}$ long, placed $5.3 \mathrm{~cm}$ in front of the $14 \mathrm{MeV}$ FNG neutron source (Figure 1). The block is composed of 12 stainless steel layers, $0.65 \mathrm{~cm}$ thick each, and $3.6 \mathrm{~cm}$ high layers (11 in total) composed of LiPb bricks, between them. Seven detector positions are planned between $3.7 \mathrm{~cm}$ and $27.7 \mathrm{~cm}$ in the block to measure the tritium production rate (TPR) using $\mathrm{Li}_{2} \mathrm{CO}_{3}$ pellets.

The modelling of the TBM HCLL mockup geometry requires full 3D description; a 2D approximate model often used in the previous studies is not applicable. Like in the previous $\mathrm{HCPB}$ tritium breeder module mockup benchmark experiment, the 3D calculational procedure was used based on the following codes:

(i) TORT [6] discrete ordinates transport code for 3D geometries,

(ii) GRTUNCL-3D [7] code to prepare uncollided and 1st collision neutron source for TORT,

(iii) SUSD3D [8] code for the cross-section sensitivity and uncertainty analysis.

SUSD3D code performs the sensitivity/uncertainty analysis and assessment of uncertainties in calculations using discrete ordinate approach based on the first-order perturbation theory where sensitivity coefficients are derived from the direct and adjoint flux moments calculated by the discrete ordinates codes. 


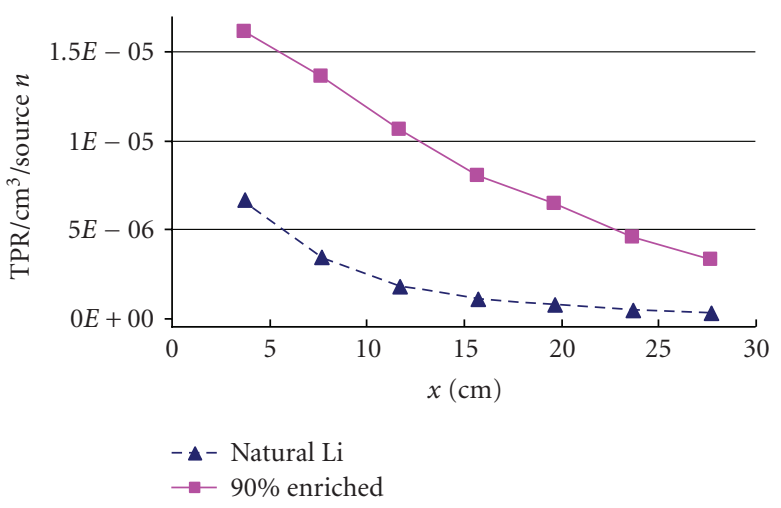

FIgURE 2: Tritium production rate for the natural and enriched Li pellets.

TABLE 1: Calculated Tritium production rates. (TORT/FENDL-2.1: S-16,P-5)

\begin{tabular}{lcc}
\hline \multicolumn{3}{c}{${ }^{6+7} \mathrm{Li}(\mathrm{n}, \mathrm{t})$ reaction rate } \\
$Y(\mathrm{~cm})$ & Natural Li & $90 \%$ enriched \\
\hline $3.7(\mathrm{P} 1)$ & $6.70 \cdot 10^{-6}$ & $1.61 \cdot 10^{-5}$ \\
$7.7(\mathrm{P} 2)$ & $3.46 \cdot 10^{-6}$ & $1.36 \cdot 10^{-5}$ \\
$11.7(\mathrm{P} 3)$ & $1.81 \cdot 10^{-6}$ & $1.06 \cdot 10^{-5}$ \\
$15.7(\mathrm{P} 4)$ & $1.08 \cdot 10^{-6}$ & $8.06 \cdot 10^{-6}$ \\
$19.7(\mathrm{P} 5)$ & $7.70 \cdot 10^{-7}$ & $6.43 \cdot 10^{-6}$ \\
$23.7(\mathrm{P} 6)$ & $4.93 \cdot 10^{-7}$ & $4.54 \cdot 10^{-6}$ \\
$27.7(\mathrm{P} 7)$ & $3.43 \cdot 10^{-7}$ & $3.30 \cdot 10^{-6}$ \\
\hline Ratio (P7/P1) & 19.5 & 4.9 \\
\hline
\end{tabular}

On the other hand, the 3D calculations are much more time consuming than the $2 \mathrm{D}$ analyses which were used in the past, for simpler benchmarks, such as the FNGITER Blanket Bulk Shield, Neutron Streaming, SiC and W benchmarks modelled in 2-dimensional geometry using the codes DORT and TWODANT. TORT calculations required several days of CPU on a Linux computer, comparing to a few minutes for 2D DORT analysis. For testing and studies of different effects and computational approximations, a simpler and approximate 2-dimensional model was therefore prepared. In the case of the HCPB tritium breeder module mockup benchmark [5] the comparison of the results obtained using the 3D code system with the results of the standard well-established 2D system based on GRTUNCL/DORT/DUSD3D codes already revealed few problems and inconsistencies in the TORT/GRTUNCL-3D code system requiring some modifications in our SUSD3D sensitivity code. Consistent use of both methods contributes in this way to better confidence in the results.

The preparation of cross-section for deterministic codes is case dependent, in particular due to theself-shielding phenomena, which are of course different than those in the previous experiments and require explicit treatment. Among the available multigroup cross-section libraries for deterministic codes, the FENDL-2 and -2.1 libraries [9] are dedicated to the fusion applications and were found the most
TABLe 2: Contribution of the uncollided flux. (TORT/FENDL-2.1: S-16,P5)

\begin{tabular}{lcc}
\hline & Uncollided and 1st collision contribution \\
$Y(\mathrm{~cm})$ & ${ }^{6+7} \mathrm{Li}(\mathrm{n}, \mathrm{t})$-natural & ${ }^{6+7} \mathrm{Li}(\mathrm{n}, \mathrm{t})-90 \%$ \\
\hline 3.7 & $56 \%$ & $6 \%$ \\
7.7 & $37 \%$ & $2 \%$ \\
11.7 & $21 \%$ & $0.8 \%$ \\
15.7 & $12 \%$ & $0.4 \%$ \\
19.7 & $8 \%$ & $0.2 \%$ \\
23.7 & $5 \%$ & $0.1 \%$ \\
27.7 & $4 \%$ & $0.1 \%$ \\
\hline
\end{tabular}

suitable. The latest version, FENDL-2.1, was used in these analyses.

The responsefunctions from IRDF-90 to the recent IRDF-2002 [10] data were processed.

The covariance matrices of lead were needed for the uncertainty analyses in addition to those already processed for the previous experiments. The most recent data were found to be available in the ENDF/B-VI.8 evaluation and were processed using the NJOY-99 code [11].

\section{RESULTS OF TRANSPORT CALCULATION}

The 3D transport calculations using the TORT code required up to several days of CPU. Both direct and adjoint calculations were performed, adjoint ones being even more time consuming ( $\sim 2$ days runs). The calculated TPR from the ${ }^{6} \operatorname{Li}(\mathrm{n}, \mathrm{t})$ and ${ }^{7} \mathrm{Li}(\mathrm{n}, \mathrm{nt})$ reactions at the 7 planned detector positions are shown in Table 1 and Figure 2. The results are consistent with those reported in [1], obtained using the MCNP Monte Carlo code. Likewise the recommendation given in [1] to use enriched Li instead of the natural one is confirmed, leading to smaller gradients between the front and back detector positions and in this way assuring that the TPR is above the detector limit for the deep detector positions.

Table 2 shows, furthermore, the fraction of the TPR produced by the direct source neutrons (uncollided flux). It is shown that the uncollided flux contribution to the TPR is important in the first detector positions in case of using natural Li. This is due to the TPR from the high energy ${ }^{7} \mathrm{Li}(\mathrm{n}, \mathrm{nt})$ reaction, as demonstrated in Table 3 presenting the ratio between the TPR from the reactions on ${ }^{6} \mathrm{Li}$ and ${ }^{7} \mathrm{Li}$. In the case of enriched Li most of tritium is as expected produced by the ${ }^{6} \mathrm{Li}(\mathrm{n}, \mathrm{t})$ reactions sensitive to thermal neutrons.

\section{RESULTS OF CROSS-SECTION SENSITIVITY/UNCERTAINTY ANALYSIS}

The cross-section sensitivity and uncertainty calculations were performed using the SUSD3D code, which requires as input quantities the direct and adjoint angular moment fluxes calculated by the discrete ordinates codes (DORT or TORT), as well as the transport cross-sections, and covariance matrices for therelevant materials and reactions. 
TABle 3: Contribution of ${ }^{6} \operatorname{Li}(n, t)$ and ${ }^{7} \operatorname{Li}(n, n t)$.

\begin{tabular}{lcc}
\hline & $\begin{array}{c}{ }^{6} \operatorname{Li}(\mathrm{n}, \mathrm{t}) /{ }^{6+7} \operatorname{Li}(\mathrm{n}, \mathrm{t}) \\
\text { Natural }\end{array}$ & $90 \%$ enriched \\
\hline 3.7 & $19 \%$ & $96 \%$ \\
7.7 & $32 \%$ & $98 \%$ \\
11.7 & $48 \%$ & $99 \%$ \\
15.7 & $62 \%$ & $99 \%$ \\
19.7 & $69 \%$ & $100 \%$ \\
23.7 & $77 \%$ & $100 \%$ \\
27.7 & $80 \%$ & $100 \%$ \\
\hline
\end{tabular}

TABLE 4: Sensitivity of the TPR to cross-sections for the detector position D7 (SUSD3D results based on the TORT 3D neutron fluxes).

\begin{tabular}{|c|c|c|c|c|c|}
\hline${ }^{6} \mathrm{Li}(\mathrm{n}, \mathrm{t})$ & & & itivity (o) & & \\
\hline Reaction & ${ }^{206} \mathrm{~Pb}$ & ${ }^{207} \mathrm{~Pb}$ & ${ }^{208} \mathrm{~Pb}$ & ${ }^{6} \mathrm{Li}$ & ${ }^{7} \mathrm{Li}$ \\
\hline Total & 0.27 & 0.25 & 0.42 & 0.96 & 0.07 \\
\hline Elastic & 0.16 & 0.16 & 0.37 & 0.004 & 0.06 \\
\hline Inelastic & 0.08 & 0.06 & 0.002 & $-5 \cdot 10^{-4}$ & \\
\hline$(\mathrm{n}, 2 \mathrm{n})$ & 0.03 & 0.02 & 0.06 & & \\
\hline$(n, 3 n)$ & $7 \cdot 10^{-5}$ & $4 \cdot 10^{-5}$ & $6 \cdot 10^{-4}$ & & \\
\hline$(\mathrm{n}, \mathrm{t})$ & & & & 0.96 & 0.01 \\
\hline$(\mathrm{n}, \gamma)$ & -0.004 & -0.003 & -0.001 & & \\
\hline Uncertainty & $2.1 \%$ & $2.0 \%$ & $6.4 \%$ & $0.2 \%$ & $0.1 \%$ \\
\hline
\end{tabular}

Both 3D and 2D sensitivity and uncertainty analyses were performed, using, respectively, fluxes calculated by the TORT code and from DORT simplified geometry calculations. Good consistency between the 2D and 3D calculations can be observed, demonstrating again that the sensitivity analyses do not require extremely precise models, which can be explained by the fact that the sensitivities are derivatives and as such relative quantities.

The sensitivities and uncertainties in the calculated TPR for two detector positions are presented in Tables 4 and 5 , calculated, respectively, using $2 \mathrm{D}$ and $3 \mathrm{D}$ computational model. The sensitivities at the $\mathrm{D} 2$ position correspond to the use of natural and $90 \%$ enriched $\mathrm{Li}$, and those at the $\mathrm{D} 7$ position are relevant both for case of natural or enriched Li.

Comparison of results in Tables 4 and 5 proves good consistency between the 2D and 3D calculations, as already mentioned above.

We can furthermore observe that the sensitivities to the $\mathrm{Pb}$ cross-sections are relatively low, below $1 \%$ of change in TPR per $1 \%$ of change in cross-section. The most sensitive reaction was found to the elastic scattering on $\mathrm{Pb}$. Controversially, this is not the reaction causing the largest uncertainty, since the corresponding cross-section uncertainty is relatively low. On the other hand, the precision in the $(n, 2 n)$ and $(n, 3 n)$ reactions on $\mathrm{Pb}$ isotopes is rather low, consequently most of the overall uncertainty in the calculated TPR is caused by the uncertainty in these crosssection reactions.
TABLE 5: Sensitivity of the TPR to cross-sections for two detector positions. Both natural and $90 \%$ enriched $\mathrm{Li}$ were considered (SUSD3D results based on the DORT 2D neutron fluxes).

(a)

\begin{tabular}{lccccc}
\hline${ }^{6+7} \mathrm{Li}(\mathrm{n}, \mathrm{t})$ & \multicolumn{5}{c}{ Sensitivity $(\% / \%)$} \\
Li-natural \\
$\begin{array}{l}\mathrm{D} 2(\sim 7 \mathrm{~cm}) \\
\text { Reaction }\end{array}$ & ${ }^{206} \mathrm{~Pb}$ & ${ }^{207} \mathrm{~Pb}$ & ${ }^{208} \mathrm{~Pb}$ & ${ }^{6} \mathrm{Li}$ & ${ }^{7} \mathrm{Li}$ \\
\hline Total & 0.02 & 0.02 & 0.01 & 0.31 & 0.69 \\
Elastic & 0.04 & 0.04 & 0.09 & $1 \mathrm{E}-3$ & 0.01 \\
Inelastic & 0.01 & 0.01 & -0.003 & $-3 \mathrm{E}-4$ & $-1 \mathrm{E}-3$ \\
$(\mathrm{n}, 2 \mathrm{n})$ & -0.03 & -0.03 & -0.08 & & \\
$(\mathrm{n}, 3 \mathrm{n})$ & $2 \cdot 10^{-5}$ & $1 \cdot 10^{-5}$ & $2 \cdot 10^{-4}$ & & \\
$(\mathrm{n}, \mathrm{t})$ & & & & 0.31 & 0.68 \\
\hline Uncertainty & $0.9 \%$ & $0.8 \%$ & $3.0 \%$ & $0.9 \%$ & $0.1 \%$ \\
\hline
\end{tabular}

(b)

\begin{tabular}{lccccc}
\hline${ }^{6+7} \mathrm{Li}(\mathrm{n}, \mathrm{t})$ & \multicolumn{5}{c}{ Sensitivity $(\% / \%)$} \\
Li-90\% enr. \\
$\begin{array}{l}\mathrm{D} 2(\sim 7 \mathrm{~cm}) \\
\text { Reaction }\end{array}$ & ${ }^{206} \mathrm{~Pb}$ & ${ }^{207} \mathrm{~Pb}$ & ${ }^{208} \mathrm{~Pb}$ & ${ }^{6} \mathrm{Li}$ & ${ }^{7} \mathrm{Li}$ \\
\hline Total & 0.29 & 0.25 & 0.50 & 0.97 & 0.06 \\
Elastic & 0.13 & 0.13 & 0.28 & $3 \cdot 10^{-3}$ & 0.04 \\
Inelastic & 0.07 & 0.05 & 0.03 & $7 \cdot 10^{-5}$ & 0.01 \\
$(\mathrm{n}, 2 \mathrm{n})$ & 0.10 & 0.08 & 0.19 & & \\
$(\mathrm{n}, 3 \mathrm{n})$ & $1 \cdot 10^{-4}$ & $6 \cdot 10^{-5}$ & $1 \cdot 10^{-3}$ & & \\
$(\mathrm{n}, \mathrm{t})$ & & & & 0.97 & 0.02 \\
\hline Uncertainty & $2.0 \%$ & $1.8 \%$ & $9.7 \%$ & $0.3 \%$ & $0.1 \%$ \\
\hline
\end{tabular}

(c)

\begin{tabular}{lccccc}
\hline${ }^{6} \mathrm{Li}(\mathrm{n}, \mathrm{t})$ & \multicolumn{5}{c}{ Sensitivity $(\% / \%)$} \\
$\mathrm{D} 7(\sim 7 \mathrm{~cm})$ & ${ }^{206} \mathrm{~Pb}$ & ${ }^{207} \mathrm{~Pb}$ & ${ }^{208} \mathrm{~Pb}$ & ${ }^{6} \mathrm{Li}$ & ${ }^{7} \mathrm{Li}$ \\
Reaction & ${ }^{20}$ & 0.27 & 0.48 & 0.96 & 0.07 \\
\hline Total & 0.31 & 0.17 & 0.37 & 0.004 & 0.06 \\
Elastic & 0.17 & 0.07 & 0.01 & $-3 \cdot 10^{-4}$ & \\
Inelastic & 0.09 & 0.04 & 0.10 & & \\
$(\mathrm{n}, 2 \mathrm{n})$ & 0.05 & $4 \cdot 10^{-5}$ & $6 \cdot 10^{-4}$ & & \\
$(\mathrm{n}, 3 \mathrm{n})$ & $7 \cdot 10^{-5}$ & & & 0.96 & 0.01 \\
$(\mathrm{n}, \mathrm{t})$ & & & & & \\
$(\mathrm{n}, \gamma)$ & -0.004 & -0.003 & -0.001 & & $0.1 \%$ \\
\hline Uncertainty & $1.9 \%$ & $1.8 \%$ & $6.5 \%$ & $0.2 \%$ & \\
\hline
\end{tabular}

Comparison of the sensitivities at the front detector positions (D2) for the natural and 90\% enriched Li in Table 5 indicates rather large differences in the sensitivities. In particular, in case of using natural $\mathrm{Li}$ in the front positions the sensitivity to the $(\mathrm{n}, 2 \mathrm{n})$ reaction on $\mathrm{Pb}$ is negative, and positive in case of using enriched as well as for deep positions (both for natural or enriched Li). This has an important and very beneficial consequence for the cross-section validation studies. Use of both enriched and natural Li would provide different (and complementary) information on the cross-sections 
and would contribute to validate the data at different energy ranges and for different nuclear reactions.

\section{CONCLUSIONS}

DORT and TORT/GRTUNCL-3D/SUSD3D computational schemes were used in the preanalysis of FNG-HCLL benchmark mock-up. The following conclusions and recommendation concerning the experimental setup can be summarised from this study.

(i) Using the ENDF/B-VI.8 covariance matrices, the uncertainties in TPR due to cross-section uncertainties were estimated to be between 5-10\%. According to these covariance data the reactions $(n, 2 n)$ and $(n, 3 n)$ on lead were found to cause the highest uncertainty, although the corresponding sensitivities are not particularly high. The TPRs are most sensitive to the $\mathrm{Pb}$ elastic scattering crosssections which are on the other hand known with much higher accuracy. Inelastic cross-sections were also found to be important for some isotopes of lead.

(ii) Large and potentially advantages differences in the sensitivities of TPR to the $\mathrm{Pb}$ cross-sections between natural and enriched Li pellets in the front detector positions were observed. This is due to the strong spectral-dependence of tritium production in different $\mathrm{Li}$ isotopes. The tritium production cross-section from ${ }^{6} \mathrm{Li}$ is high at low energies, while tritium production in ${ }^{7} \mathrm{Li}$ is a threshold reaction. In particular, in the front positions the sensitivity to the $(n, 2 n)$ reaction on $\mathrm{Pb}$ is negative in case of using natural $\mathrm{Li}$, and positive in case of using enriched Li. For deep positions the sensitivity is positive both for natural or enriched Li. This has an important and very beneficial consequence for the cross-section validation studies. Use of both enriched and natural Li would provide complementary information on the cross-sections and would permit to validate the data at different energy ranges and for different nuclear reactions. Combined use of natural and enriched Li pellets is therefore strongly recommended for the TPR measurements at the front detector positions, as far as the neutron flux level (and the experimental setup) permits it.

(iii) In addition to measurements on the axis of the experimental setup, off-axis measurements would provide additional information on the angular distributions, as well as on TPR generated at different energy spectra. The feasibility of placing the detectors at off-axis positions in the experimental setup should be investigated.

\section{ACKNOWLEDGMENTS}

This work was carried out in support of the SINBAD project of the OECD/NEA Data Bank, in the framework of the European Fusion Development Agreement and was in part financed by the EC of the EURATOM/ENEA/FzK/IJS contract.

\section{REFERENCES}

[1] P. Batistoni, "Design of a HCLL Mock-up for Neutronics Studies,” FUS TEC MA-NE-R-015, December 2005.
[2] P. Batistoni and R. Villari, "Task TTMN-002, pre-analysis of the TBM-HCLL benchmark experiment," in Proceedings of the JEFF/EFF Working Group Meeting, Paris, France, May 2006.

[3] I. Kodeli, "Benchmark experiments collection and analysis: news on the SINBAD project; FNG-HCLL benchmark preanalysis," in Proceedings of the EFF Meeting, November 2006, OECD/NEA, EFF-DOC-996.

[4] P. Batistoni, M. Angelone, L. Bettinali, et al., "Neutronics experiment on a helium cooled pebble bed (HCPB) breeder blanket mock-up," Fusion Engineering and Design, vol. 82, no. 15-24, pp. 2095-2104, 2007.

[5] I. Kodeli, "Deterministic 3D transport, sensitivity and uncertainty analysis of TPR and reaction rate measurements in HCPB breeder blanket mock-up benchmark," in Proceedings of the International Conference on Nuclear Energy for New Europe, Portorož, Slovenia, September 2006.

[6] W. A. Rhoades and R. L. Childs, "DOORS 3.2, one-, two-, three-dimensional discrete ordinates neutron/photon transport code system," Tech. Rep. CCC-650, Radiation Safety Information Computational Center, Oak Ridge National Laboratory, Oak Ridge, Tenn, USA, 1998.

[7] J. O. Johnson, Ed., "GRTUNCL3D, code to calculate semianalytic first collision source and uncollided flux," Tech. Rep., Oak Ridge National Laboratory, Oak Ridge, Tenn, USA, July 2004.

[8] I. Kodeli, "Multidimensional deterministic nuclear data sensitivity and uncertainty code system: method and application," Nuclear Science and Engineering, vol. 138, no. 1, pp. 45-66, 2001.

[9] H. Wienke and M. Herman, "FENDL/MG-2.0 and FENDL/ MC-2.0, the processed cross-section libraries for neutronphoton transport calculations, version 1," Tech. Rep. IAEANDS-176 Rev. 1, International Atomic Energy Agency, Vienna, Austria, February 1998.

[10] International Atomic Energy Agency, International Reactor Dosimetry File 2002 (IRDF-2002), Technical Reports Series, no. 452, International Atomic Energy Agency, Vienna, Austria, 2006.

[11] R. E. MacFarlane and D. W. Muir, "The NJOY nuclear data processing system,” Version 97, LA-12740-M, 1994. 

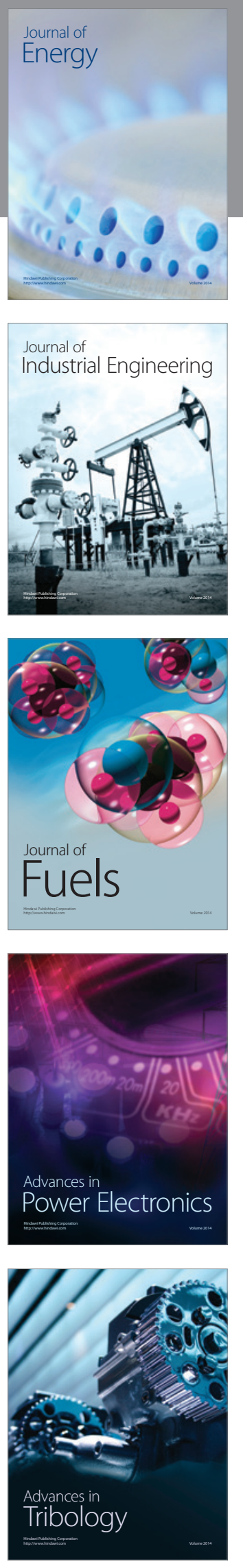
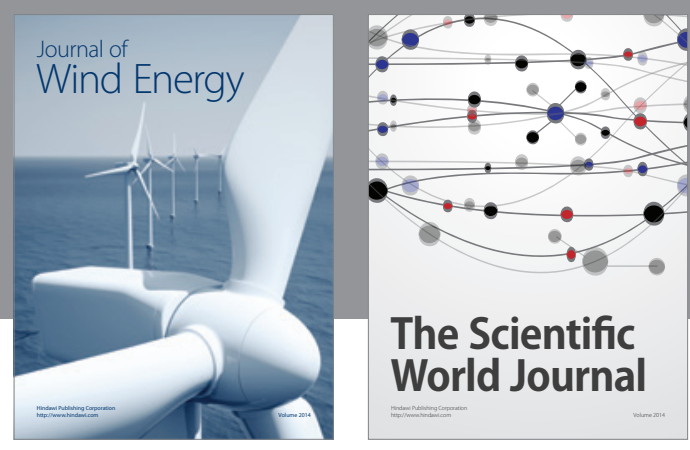

The Scientific World Journal

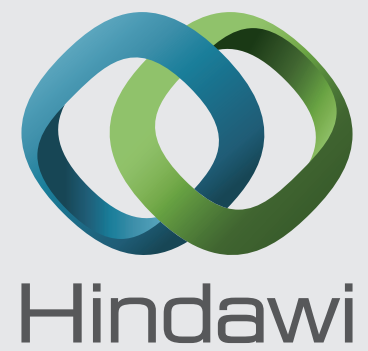

Submit your manuscripts at http://www.hindawi.com
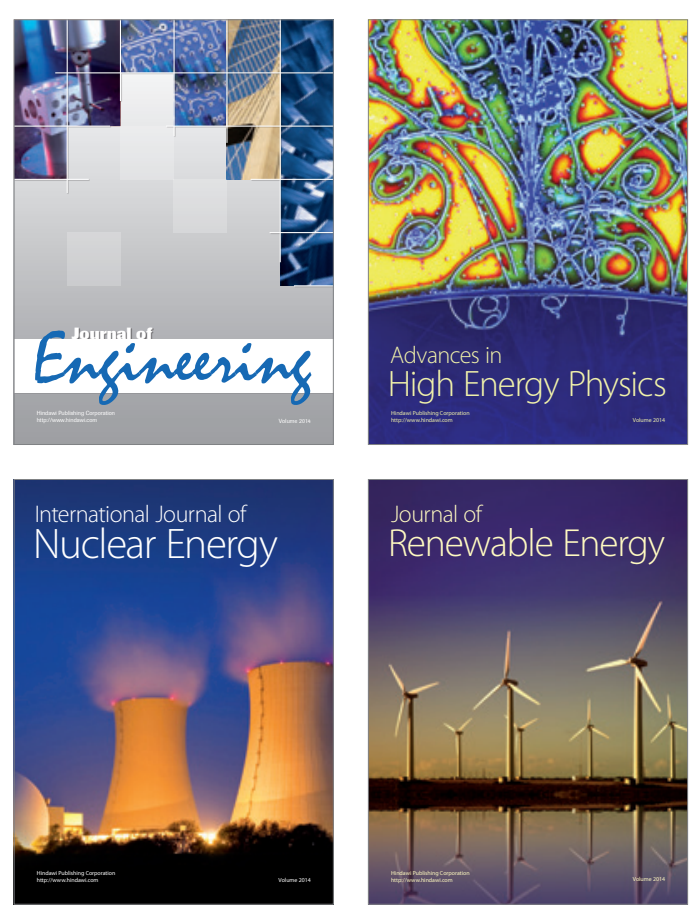

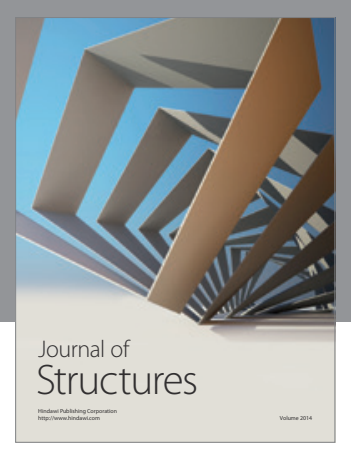

Rotating
Mechinery
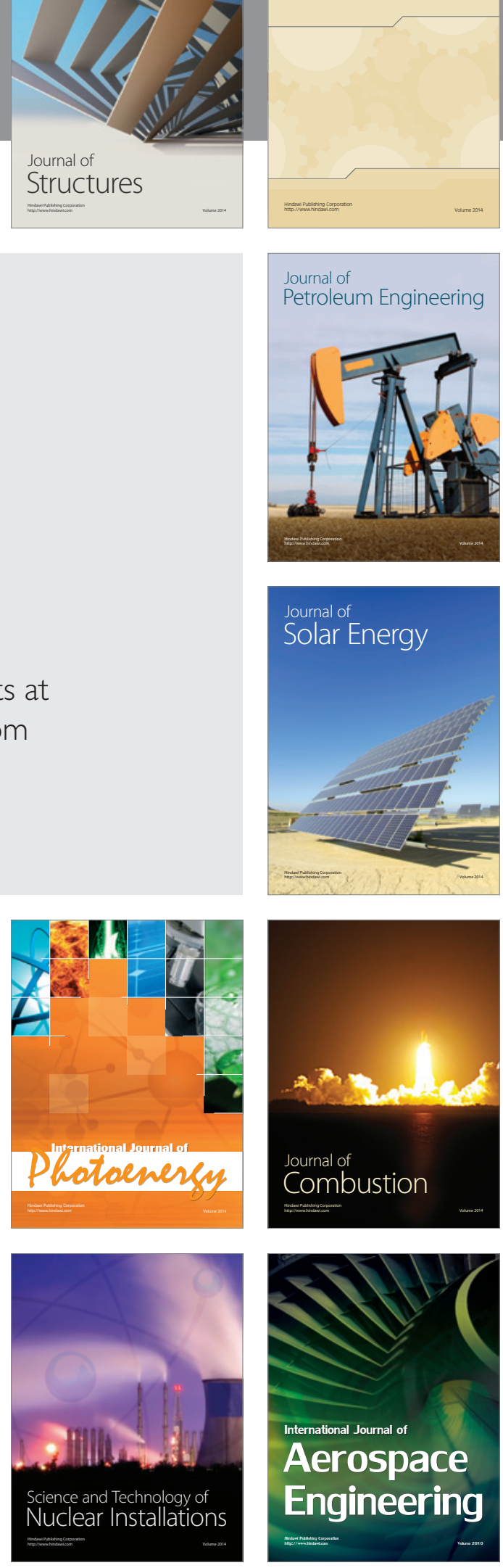